\title{
Photodissociation dynamics of the LiF molecule: Two- and three-state descriptions
}

\author{
A. Tóth, ${ }^{1, *}$ A. Csehi, ${ }^{2,1}$ G. J. Halász, ${ }^{3}$ and Á. Vibók ${ }^{2,1, \dagger}$ \\ ${ }^{1}$ ELI-ALPS, ELI-HU Non-Profit Ltd, H-6720 Szeged, Dugonics tér 13, Hungary \\ ${ }^{2}$ Department of Theoretical Physics, University of Debrecen, H-4010 Debrecen, PO Box 5, Hungary \\ ${ }^{3}$ Department of Information Technology, University of Debrecen, H-4010 Debrecen, PO Box 12, Hungary
}

(Received 31 October 2018; published 24 April 2019)

\begin{abstract}
The photodissociation of the lithium fluoride molecule gathered much attention lately. Theoretical works treating this subject usually consider the $1{ }^{1} \Sigma^{+}$and $2{ }^{1} \Sigma^{+}$electronic states, which are coupled nonadiabatically. As a continuation of a previous work, we intend to highlight the importance of the $1^{1} \Pi$ state by investigating the kinetic energy and angular distribution of the photofragments. Besides, our results pointed out the importance of the molecular rotation.
\end{abstract}

DOI: 10.1103/PhysRevA.99.043424

\section{INTRODUCTION}

Understanding the behavior of atoms and molecules in a laser field is an intensively studied area. There are a huge amount of theoretical and experimental works available which have discussed numerous new phenomena of light-matter interaction such as high harmonic generation [1,2], above threshold ionization [3], dissociation [4], bond softening and hardening effects [5-9], etc. Many of these works tackle the dynamical problems of diatomic systems, starting investigations from the simplest hydrogenlike ions or molecules (see, for example, Refs. [10-14] and references therein) to manyelectron objects [15-24].

The bond hardening or vibrational trapping effect can be well understood using the dressed state or light-induced potential (LIP) representation which involves the nuclear degree of freedom (most commonly the molecular vibration) in the treatment of diatomic molecules [25,26]. This phenomenon can also be named as light-induced avoided crossing (LIAC) $[27,28]$. By further extending the model and incorporating a second degree of freedom into the theoretical description one can arrive to the so-called "light-induced conical intersection" (LICI) situation [27,28]. The rotational coordinate can serve as a second degree of freedom as the laser rotates the molecule. It has been demonstrated that similarly to the natural conical intersection (CI) situation [29-33], the LICI gives rise to a variety of nonadiabatic phenomena, as well. Theoretical and experimental studies have pointed out that the LICIs have significant impact on the different dynamical properties (like molecular spectra, molecular alignment, or photodissociation probability, etc.) of diatomic [14,34-40] and polyatomic molecules [41-43].

In one of our former works [44] we have studied the LiF molecule which belongs to the family of alkali-metal halides. There have been a vast number of theoretical works dealing with the photodissociation problem of this molecule (see,

\footnotetext{
*Attila.Toth@eli-alps.hu

†Vibok@phys.unideb.hu
}

for example, Refs. [44-53] and references therein). During our investigations [44] we emphasized the role played by the lowest-lying $\Pi$ electronic state in the photodissociation process of the molecule discussing the population dynamics. Among others, we compared results obtained treating only the vibrational motion (LIAC situation) with ones also considering the rotation (LICI situation) of the molecule placed in an off-resonant continuous wave (CW) laser field. In the latter case we found a suppression of the recently reported [53] wave-packet confinement effect.

This paper is devoted to further investigate the dynamics of the system under the action of an off-resonant driving field and provide a deeper understanding of the undergoing processes analyzing the angular distribution and the kinetic energy release (KER) spectra of the photofragments.

The article is arranged as follows. In the next section, we present the background required for our theoretical study. Here, we discuss the working Hamiltonian, the time-dependent electric field of the applied laser pulses, the physical quantities under investigation, and the method used to compute the propagation of the quantum wave packets employing the working Hamiltonian. The third section is dedicated to present the results and discussion of the kinetic energy release spectra (KER) and the angular distribution of the photofragments. Finally, the last section summarizes our findings.

\section{PHYSICAL SITUATION AND METHODS}

During our numerical simulations, the LiF molecule is modeled either as a three-level system employing the three lowest-lying singlet electronic states, namely, $1^{1} \Sigma^{+}, 2{ }^{1} \Sigma^{+}$ and $1^{1} \Pi^{+}$(labeled throughout the paper as $\Sigma_{1}, \Sigma_{2}$, and $\Pi_{1}$ ) or a two-level system, where only the $1^{1} \Sigma^{+}$and $2{ }^{1} \Sigma^{+}$ states are considered. The corresponding potential energy curves are presented in Fig. 1(a). An important feature of the system is the avoided crossing (AC) between the $V_{\Sigma_{1}}$ and $V_{\Sigma_{2}}$ potential curves around $R \sim 7.2 \AA$. The corresponding intrinsic nonadiabatic coupling term $\left[\tau(R)=\left\langle\varphi_{\Sigma_{1}} \mid \frac{\partial}{\partial R} \varphi_{\Sigma_{2}}\right\rangle\right]$ is also plotted here. Figures 1(b) and 1(c) present the 


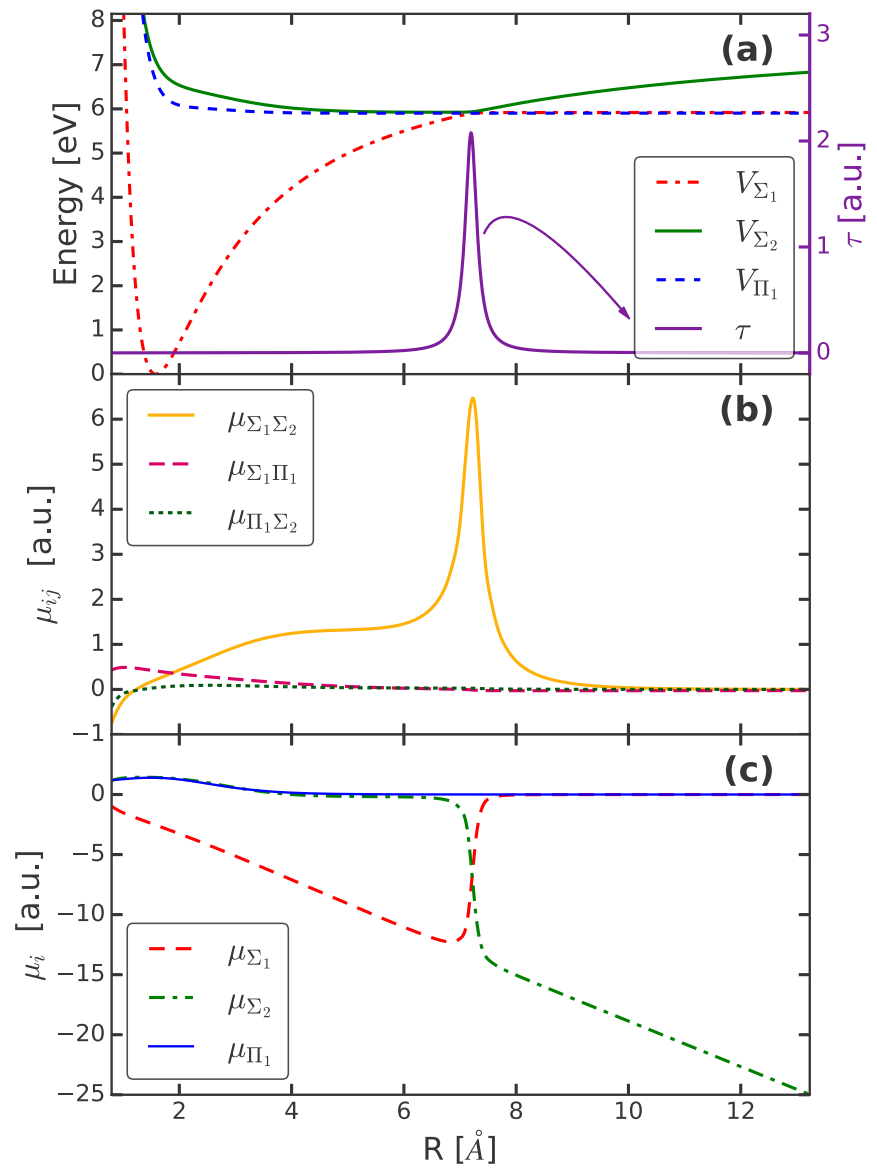

FIG. 1. (a) The lowest three adiabatic potential energy curves of the LiF molecule and the nonadiabatic coupling term $\tau(R)$ between the two $\Sigma$ states (scale is on the right side). (b) The transition dipole moment functions between the different electronic states. (c) Permanent dipole moment functions of the three adiabatic electronic states.

transition $\left[\mu_{i j}(R)=-\left\langle\varphi_{i}\left|\sum_{k} r_{k}\right| \varphi_{j}\right\rangle\right]$ and the permanent $\left[\mu_{i}(R)=-\left\langle\varphi_{i}\left|\sum_{k} r_{k}\right| \varphi_{i}\right\rangle\right]$ dipole moments $\left(i, j \in\left\{\Sigma_{1}, \Sigma_{2}\right.\right.$, $\left.\left.\Pi_{1}\right\}\right)$, respectively. An essential difference among the transition dipole moments (TDM) is that $\vec{\mu}_{\Sigma_{1} \Sigma_{2}}$ is parallel with the molecular axis while the ones corresponding to the $\Sigma-\Pi$ transitions are perpendicular. In contrast, all permanent dipole moments (PDM) are parallel with the molecule.

Computation of the above electronic structure quantities of $\mathrm{LiF}$ have been carried out with the MOLPRO [54] program package at the MRCI/CAS(6/12)/aug-cc-pVQZ level of theory. In particular, the $\tau(R)$ has been computed by finite differences of the MRCI electronic wave functions. The number of active electrons and molecular orbitals in the individual irreducible representations of the $C_{2 v}$ point group were $A_{1} \rightarrow 2 / 5, B_{1} \rightarrow 2 / 3, B_{2} \rightarrow 2 / 3, A_{2} \rightarrow 0 / 1$. With these parameters, we could achieve a good agreement with the results of other studies $[55,56]$.

Theoretical works found in the literature almost exclusively treat the LiF molecule as a two-level system, considering only the two lowest-lying $\Sigma$ states. Figure 1 shows, however, that the $\Pi_{1}$ state is energetically very close to the $\Sigma_{2}$ state, making it indispensable when interpreting experimental data. Moreover, as both the $\mu_{\Sigma_{1} \Sigma_{2}}$ and $\mu_{\Sigma_{1} \Pi_{1}}$ are nonzero around the Franck-Condon region, it is expected that both the $\Sigma_{2}$ and $\Pi_{1}$ states to be populated by a suitably tuned short laser pulse. These observations motivated us to investigate the effect of the $\Pi_{1}$ state on various experimentally measurable physical quantities of the photodissociation process.

\section{A. Working Hamiltonian}

To describe realistically the LiF molecule, all dipole moments mentioned above are included in the calculations. Accordingly, the time-dependent nuclear Hamiltonian for the three-state system reads as

$$
\begin{aligned}
\hat{H}= & \left(-\frac{1}{2 M_{r}} \frac{\partial^{2}}{\partial R^{2}}+\frac{L_{\theta}^{2}}{2 M_{r} R^{2}}\right) \cdot \mathbf{1}+\left(\begin{array}{ccc}
V_{\Sigma_{1}} & 0 & K \\
0 & V_{\Pi_{1}} & 0 \\
-K & 0 & V_{\Sigma_{2}}
\end{array}\right) \\
& -E(t)\left(\begin{array}{ccc}
\mu_{\Sigma_{1}} \cos (\theta) & \mu_{\Sigma_{1} \Pi_{1}} \sin (\theta) & \mu_{\Sigma_{1} \Sigma_{2}} \cos (\theta) \\
\mu_{\Sigma_{1} \Pi_{1}} \sin (\theta) & \mu_{\Pi_{1}} \cos (\theta) & \mu_{\Pi_{1} \Sigma} \sin (\theta) \\
\mu_{\Sigma_{1} \Sigma_{2}} \cos (\theta) & \mu_{\Pi_{1} \Sigma_{2}} \sin (\theta) & \mu_{\Sigma_{2}} \cos (\theta)
\end{array}\right) .
\end{aligned}
$$

Here, the first term describes the kinetic energy of the system with $R$ and $\theta$ (the angle between the laser polarization direction and the molecular axis) being the vibrational and rotational coordinates, respectively. The 1 denotes the $3 \times 3$ unit matrix, $M_{r}$ is the reduced mass, while $L_{\theta}$ is the angular momentum operator with $m=0$. The second term contains the potentials of the electronic states and the intrinsic nonadiabatic coupling operator $K$ associated with the $\Sigma_{1}-\Sigma_{2}$ avoided crossing. We employed an approximated form of this operator $K(R) \approx \frac{1}{2 M_{r}}\left[2 \tau(R) \frac{\partial}{\partial R}+\frac{\partial}{\partial R} \tau(R)\right.$ ] [57], with $\tau$ being the nonadiabatic coupling term presented on Fig. 1(a). The last term in the expression of $\hat{H}$ describes the laser-matter interaction in the dipole approximation with the PDMs on the diagonal and the TDMs in the off-diagonal positions of the dipole matrix, while $E(t)$ represents the applied laser field. In case when only the $\Sigma_{1}$ and $\Sigma_{2}$ states are considered, i.e., the two-state description, all terms related to the $\Pi_{1}$ state are dropped from the working Hamiltonian. Unless specified otherwise, atomic units with $e=m_{e}=\hbar=1$ are used throughout the article.

Equation (1) describes the three-state rotating-vibrating molecules, hence, the calculations involving it are named three-state two-dimensional (2D) simulations. We also investigated the case when the rotational motion is frozen leading to the so-called one-dimensional (1D) simulations. In this case, the $\theta$ dynamical variable of Eq. (1) becomes a parameter, and for obtaining a correct comparison with the $2 \mathrm{D}$ results an averaging over numerous calculations for the different $\theta$ values is performed. There is a significant difference between the two descriptions. As a result of the noncrossing rule, the 1D approach which only takes into account the single internal vibrational degree of freedom of the diatomic molecule is able to represent light-induced avoided crossings (LIACs). In contrast, treating the angle $\theta$ as a dynamical variable provides the second degree of freedom necessary for the twodimensional "branching" space in which light-induced conical intersections (LICIs) can exist. 


\section{B. Floquet description}

All the results presented in this paper were obtained by solving the time-dependent Schrödinger equation written for the Hamiltonian of Eq. (1) (and its variants, i.e., two-state and 1D). For an easier interpretation of these results we turned to the Floquet theory $[58,59]$ which is widely used to explain various strong field phenomena. This method is best suited for the investigation of periodically timedependent systems, which is the case for our driving field

$$
\hat{V}_{\text {Floquet }}=\left(\begin{array}{ccc}
\ddots & \vdots & \vdots \\
\cdots & \hat{V}_{0}-2 \omega_{d} & \hat{\hat{D}} \\
\cdots & \hat{D}^{\dagger} & \hat{V}_{0}-1 \omega_{d} \\
\cdots & 0 & \hat{D}^{\dagger} \\
\cdots & 0 & 0 \\
\cdots & 0 & 0 \\
. \cdot & \vdots & \vdots
\end{array}\right.
$$

This Floquet potential matrix has a tridiagonal form with the electronic state energies on the diagonal

$$
\hat{V}_{0}=\left(\begin{array}{ccc}
V_{\Sigma_{1}} & 0 & 0 \\
0 & V_{\Pi_{1}} & 0 \\
0 & 0 & V_{\Sigma_{2}}
\end{array}\right),
$$

while the permanent dipole and the dipole coupling matrix elements between the different electronic states in the offdiagonal blocks

$$
\hat{D}=\frac{E_{0}}{2}\left(\begin{array}{ccc}
\mu_{\Sigma_{1}} \cos (\theta) & \mu_{\Sigma_{1} \Pi_{1}} \sin (\theta) & \mu_{\Sigma_{1} \Sigma_{2}} \cos (\theta) \\
\mu_{\Sigma_{1} \Pi_{1}} \sin (\theta) & \mu_{\Pi_{1}} \cos (\theta) & \mu_{\Pi_{1} \Sigma 2} \sin (\theta) \\
\mu_{\Sigma_{1} \Sigma_{2}} \cos (\theta) & \mu_{\Pi_{1} \Sigma_{2}} \sin (\theta) & \mu_{\Sigma_{2}} \cos (\theta)
\end{array}\right),
$$

with $E_{0}$ being the amplitude of the considered electric field. $\hat{V}_{\text {Floquet }}$ has a periodic structure with different number $\left(n_{p h}\right)$ of $\omega_{d}$ in the individual diagonal blocks. Diagonalizing this matrix leads to the adiabatic or light-induced potentials (LIPs) that proved to be useful in understanding the dynamics of the system.

As an example, on Fig. 2 we present the LIPs obtained for the highest considered driving field intensity $\left(I_{d}=4 \times\right.$ $10^{13} \mathrm{~W} / \mathrm{cm}^{2}$ ) for both the two- and three-state descriptions. The calculations were performed on the range of 0.8-4.0 $\AA$ A internuclear distances since the expression for the Floquet potential matrix is only valid far away from the intrinsic avoided crossing, and with $n_{p h}=-5, \ldots, 5$, hence accounting for multiphoton processes. We found that with increasing field intensity, the LIPs deviate from being just copies of the uncoupled potential surfaces shifted with $\omega_{d}$, but they hybridize in the regions close to resonances. Aside from the shape of the LIPs this is illustrated also by the coloring of the surfaces, which codifies the diabatic states from which they originate, and was achieved using the eigenvectors of $\hat{V}_{\text {Floquet }}$ that form the transformation matrix. As on Fig. 1(a), red, green, and blue colors correspond to the $\Sigma_{1}, \Sigma_{2}$, and $\Pi_{1}$ states, respectively. (see Sec. IIC), and is based on the transformation of the Schrödinger equation into an equivalent infinite-dimensional time-independent matrix eigenvalue problem. We do not intend to apply it as an alternative for the numerical exact propagation, but use it to provide an illustrative picture of the energy landscape of the LiF molecule under the action of a CW laser field. For this reason, when building our Floquet matrix we neglected the kinetic energy terms of the Hamiltonian:

$\left.\begin{array}{cccc}\vdots & \vdots & \vdots & . \cdot \\ 0 & 0 & 0 & \cdots \\ \hat{D} & 0 & 0 & \cdots \\ \hat{V}_{0} & \hat{D} & 0 & \cdots \\ \hat{D}^{\dagger} & \hat{V}_{0}+1 \omega_{d} & \hat{D} & \cdots \\ 0 & \hat{D}^{\dagger} & \hat{V}_{0}+2 \omega_{d} & \cdots \\ \vdots & \vdots & \vdots & \ddots\end{array}\right)$

\section{Applied electric field}

The laser field $E(t)$ employed in our calculations is the sum of two linearly polarized (in the same direction) components:

$$
E(t)=E_{p} f_{p}(t) \cos \left[\omega_{p}\left(t-t_{0}\right)\right]+E_{d} f_{d}(t) \cos \left(\omega_{d} t\right) .
$$

The first term represents a pump pulse of energy $\omega_{p}$ and amplitude $E_{p}$, which initiates the dynamics of the system by transferring part of the initial population from the ground to the excited states. We chose to employ cosine-square-shaped pulses, i.e., the envelope function is given by

$$
f_{p}(t)=\cos ^{2}\left(\frac{1.14372\left(t-t_{0}\right)}{\tau_{p}}\right),
$$

where $\tau_{p}$ is the full width at half-maximum (FWHM) of the intensity profile of the pulse. In the above equations, $t_{0}$ indicates the center of the pulse, which was chosen to coincide with the origin of our time axis, that is, $t_{0}=0$. For all the results presented in this work, the energy of the pump pulse was fixed to $\omega_{p}=6.94 \mathrm{eV}$, its intensity to $I_{p}=4.8 \times 10^{13} \mathrm{~W} / \mathrm{cm}^{2}$, and duration to $\tau_{p}=20 \mathrm{fs}$.

The second term of Eq. (5) represents the continuous wave driving field. The energy of this field was set to $\omega_{d}=$ $3.995 \mathrm{eV}$ which is unable to produce resonant transitions from the ground state to the excited ones, therefore it only modifies the molecules' environment. Several different intensity values were used ranging from $I_{d}=1.69 \times 10^{12} \mathrm{~W} / \mathrm{cm}^{2}$ to $I_{d}=4 \times$ $10^{13} \mathrm{~W} / \mathrm{cm}^{2}$. The required maximal electric field amplitude was reached linearly from zero over a 500-fs-long ramp-up period prior to the pump pulse, which was necessary to avoid undesired state mixings produced by a nonadiabatic sudden switch on. After this buildup period, the driving intensity remained constant.

\section{Propagation of the wave packets}

The time-dependent Schrödinger equation (TDSE) that described the dynamics of the system was solved using the MCTDH (multiconfigurational time-dependent Hartree) method [60-62]. The vibrational degree of freedom $(R)$ was described by a sin-DVR primitive basis with $N_{R}$ basis 

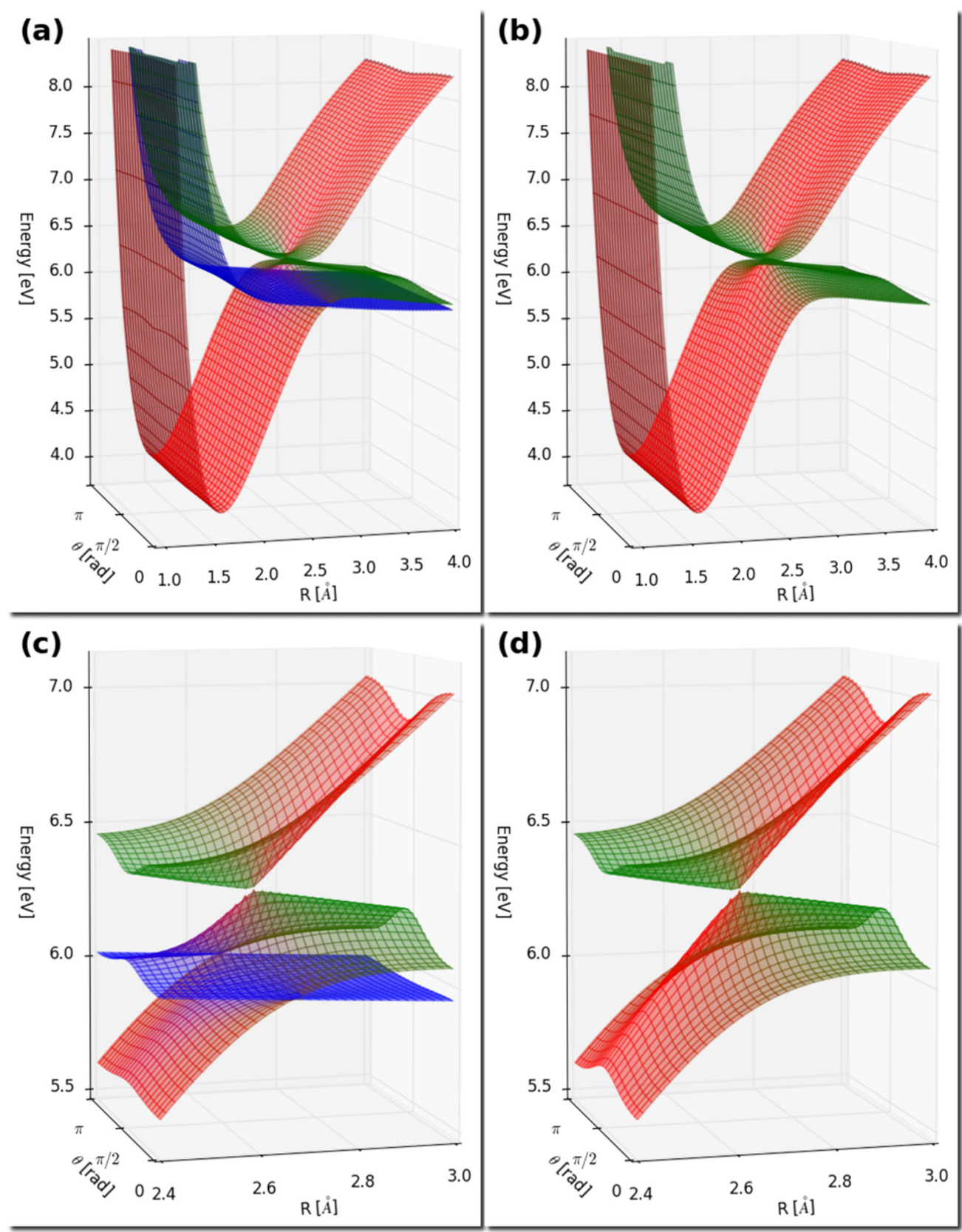

FIG. 2. Light-induced potential energy surfaces for the three-state (a), (c) and two-state (b), (d) models calculated for the highest driving field intensities $I_{d}=4 \times 10^{13} \mathrm{~W} / \mathrm{cm}^{2}$ considered in this work. (c), (d) Show a zoom of the LIPs presented on (a) and (b), respectively, and concentrating on the LIAC (LICI) and regions. The coloring reflects the diabatic states that make up the different points of the adiabatic surfaces: red, $\Sigma_{1}$; blue, $\Pi_{1}$; green, $\Sigma_{2}$.

elements distributed between 0.79 and $31.74 \AA$ for the internuclear separation. For the description of the rotational degree of freedom $(\theta)$ Legendre polynomials $\left\{P_{J}(\cos \theta)\right\}_{j=0,1,2, \ldots, N_{\theta}}$ were used. These primitive basis sets $(\chi)$ were employed to represent the single-particle functions $(\phi)$, which in turn were used to build up the nuclear wave function $(\psi)$ :

$$
\begin{aligned}
\phi_{j_{q}}^{(q)}(q, t) & =\sum_{l=1}^{N_{q}} c_{j_{q} l}^{(q)}(t) \chi_{l}^{(q)}(q), \quad q=R, \theta \\
\psi(R, \theta, t) & =\sum_{j_{R}=1}^{n_{R}} \sum_{j_{\theta}=1}^{n_{\theta}} A_{j_{R}, j_{\theta}}(t) \phi_{j_{R}}^{(R)}(R, t) \phi_{j_{\theta}}^{(\theta)}(\theta, t) .
\end{aligned}
$$

In our numerical calculations, $N_{R}=2048$ and $N_{\theta}=271$ primitive basis functions were used. On all adiabatic surfaces and for both degrees of freedom, a set of $n_{R}=n_{\theta}=15,20,30$, and 35 single-particle functions were used to build up the nuclear wave function of the system. The actual values of $n_{R}=n_{\theta}$ were chosen depending on the peak intensity of the applied laser fields in such a way to ensure the correct convergence of all propagations.

\section{E. Calculated quantities}

The solutions of the TDSE were then used to calculate the populations of the employed electronic states [44], the kinetic 
energy release spectra (KER), and the angular distribution of the molecular fragments [14]. The electronic state populations are obtained as

$$
\begin{aligned}
P_{i}(t)= & \left\langle\psi_{i}(R, \theta, t) \mid \psi_{i}(R, \theta, t)\right\rangle \\
= & \int_{0}^{\pi} d \theta \sin \theta \int_{0}^{\infty} d R \psi_{i}^{*}(R, \theta, t) \psi_{i}(R, \theta, t), \\
& i \in\left\{\Sigma_{1},\left(\Pi_{1}\right), \Sigma_{2}\right\}
\end{aligned}
$$

where $\psi_{i}$ are the projections of the total nuclear wave function of Eq. (7) on the considered electronic states. The KER is calculated according to the following formula:

$$
P_{\mathrm{KER}}^{i}(E)=\int_{0}^{\infty} d t \int_{0}^{\infty} d t^{\prime}\left\langle\psi_{i}(t)|W| \psi_{i}\left(t^{\prime}\right)\right\rangle e^{-i E\left(t-t^{\prime}\right)},
$$

where $-i W$ is the complex absorbing potential (CAP) applied at the last $5.29 \AA$ of the grid related to the vibrational degree of freedom of each electronic state. The angular distribution of the photofragments is given by

$$
P_{\text {ang }}^{i}\left(\theta_{j}\right)=\frac{1}{w_{j}} \int_{0}^{\infty} d t\left\langle\psi_{i}(t)\left|W_{\theta_{j}}\right| \psi_{i}(t)\right\rangle,
$$

where $-i W_{\theta_{j}}$ is the projection of the CAP to a specific direction of the angular grid $\left(j=0, \ldots, N_{\theta}\right)$, and $w_{j}$ is the DVR weight associated to this grid point. In the last two equations, the superscript $i$ stands for either $\Sigma_{1}$ or $\Pi_{1}$ as the molecule can dissociate on these two states.

\section{RESULTS AND DISCUSSION}

Before proceeding to the analysis of the kinetic energy release spectra and angular distribution of the photofragments, let us have an overview of the systems behavior. In the calculations we assumed initially unaligned molecules. The initial nuclear wave packet ( $\left.\Psi_{\text {init }}\right)$ of this isotropic distribution was in its rotational ground state $(J=0)$ and in the vibrational ground state $(v=0)$ of the $\Sigma_{1}$ electronic state. The dynamics of the system is initiated by the pump pulse, which promotes part of the initial population from the ground state $\left(\Sigma_{1}\right)$ to the excited ones $\left(\Pi_{1}\right.$ and/or $\left.\Sigma_{2}\right)$. Based on the analysis performed on the evolution of the electronic state populations in our former work (see Fig. 3 of Ref. [44]), in the absence of a continuous wave driving field the excited wave packets start moving toward larger internuclear separations and finally dissociate. Since $\Sigma_{2}$ is a bound state, dissociation is achieved through a population transfer to the $\Sigma_{1}$ state at the intrinsic avoided crossing present in the system. However, not all the excited population gets transferred during the first passage through the AC region, and a small part of it proceeds to oscillate on the $\Sigma_{2}$ surface. Due to the shallow nature of the $\Sigma_{2}$ potential, this oscillation has a long period $(\approx 800 \mathrm{fs})$ and we did not propagate until the complete dissociation of the system, but performed our calculation only up to $t_{\text {final }}=1000 \mathrm{fs}$. If a driving field was applied, some oscillations appeared in the population curves. These oscillations increased with increasing driving field intensity and were more pronounced for the $1 \mathrm{D}$ calculations. At the same time, the dissociation yield dropped.

In what follows, we are looking deeper into the details of the above-outlined dynamics and try to explain the differences obtained in case of the 1D and 2D calculations, and the impact of the $\Pi_{1}$ state on the dynamical behavior of the system.

\section{A. Kinetic energy release spectra}

Let us start by investigating the kinetic energy release (KER) spectra of the dissociation products. These spectra are presented on Fig. 3, where the left and right columns group the outcome of the 1D and the 2D calculations, respectively. The different rows show the results obtained for different driving field intensities in the interval of $I_{d}=$ $1.69 \times 10^{12} \mathrm{~W} / \mathrm{cm}^{2}-4 \times 10^{13} \mathrm{~W} / \mathrm{cm}^{2}$. As mentioned earlier, dissociation from the bound $\Sigma_{2}$ state is achieved through a population transfer at the intrinsic AC to the $\Sigma_{1}$ state, therefore, the KER is only calculated for the $\Sigma_{1}$ [marked with red $(+)$ and green lines $(x)$ for the three-state and two-state models, respectively] and the $\Pi_{1}$ [blue (o) curves] states.

In the absence of a driving field, presented on Figs. 3(a) and 3(b), the spectrum of the two-state calculations shows a featureless Lorentzian profile centered at $E_{0}=E_{\nu=0}+\omega_{p}$ marked with magenta arrows on the figures. This is easily understood based on the dynamics mentioned earlier. At the chosen pump energy of $\omega_{p}=6.94 \mathrm{eV}$, the resonant coupling between the two states occurs near the center of the initial wave packet. The spectrally broad 20 -fs-long pump pulse creates an excited wave packet on the $\Sigma_{2}$ state with a similarly wide energy distribution, which is largely transferred back to the $\Sigma_{1}$ state at the AC, and later dissociates.

Figures 3(a) and 3(b) also show that including the $\Pi_{1}$ state in the calculations has a significant impact on the energy distribution of the photofragments. The spectra of the $\Sigma_{1}$ state are shifted toward higher energies, while the $P_{\mathrm{KER}}^{\Pi_{1}}$ is split into two peaks. A superficial analysis would expect $P_{\mathrm{KER}}^{\Pi_{1}}$ to have a similar shape as $P_{\mathrm{KER}}^{\Sigma_{1}}$, but centered at slightly smaller energies than $E_{0}$ as the center of the initial wave packet is located at larger internuclear distances than the position of resonant coupling. This behavior was observed in results obtained for smaller pump-pulse intensities, but not presented in this paper. However, for the present pulse parameters, there is a strong competition between the $\Sigma_{1} \rightarrow \Sigma_{2}$ and $\Sigma_{1} \rightarrow \Pi_{1}$ transfer processes. As mentioned earlier, the position of the resonant coupling to the $\Sigma_{2}$ state is near the center of the initial wave packet, while resonance with the $\Pi_{1}$ state occurs farther away. Also considering that over the region where the initial wave packet $\Psi_{\text {init }}$ is nonzero, $\mu_{\Sigma_{1} \Pi_{1}}$ is larger than $\mu_{\Sigma_{1} \Sigma_{2}}$, the shape of the spectra becomes understandable: the middle part of $\Psi_{\text {init }}$ "feeds" the $\Sigma_{2}$ state while its edges populate $\Pi_{1}$ and as the spectrally broad pump pulse covers the entire region, we end up with the two peaks for $\Pi_{1}$. The motive for the competition of the pump processes is that already during the leading edge of the pump pulse the population transferred to the excited states exceeds that on the ground state. Furthermore, as the laser intensity increases, multiphoton processes start to occur, which reverse the population transfer toward the $\Sigma_{1}$ state, resulting in a back and forth mixing of the populations. Accordingly, the shift of the spectra toward higher energies can be attributed to the fact that during the mixing the wave packets on the excited states start to move toward larger internuclear distances, hence acquiring some kinetic energy. Comparing the 1D [Fig. 3(a)] 


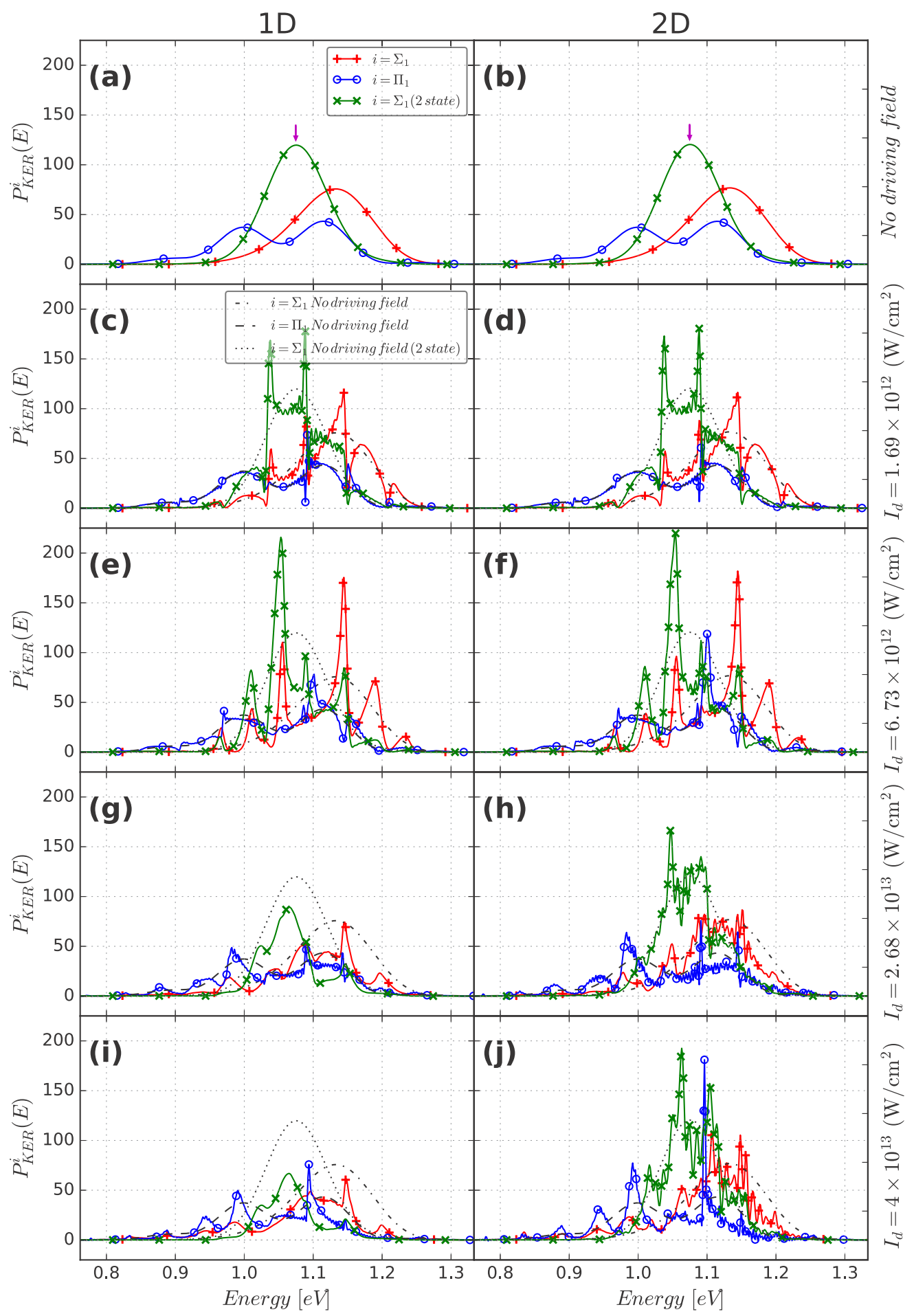

FIG. 3. Kinetic energy release spectra of the photofragments for the considered driving field intensities (indicated on the right axis of each row). On all panels, red lines with + symbols and blue lines with o indicate the spectra obtained for the three-state calculations on the $\Sigma_{1}$ and $\Pi_{1}$ states, respectively. The green lines marked with $\times$ show the results of the two-state calculations. Broken black lines mark the spectra obtained without a driving field.

and 2D [Fig. 3(b)] results we can see that they are practically identical, meaning that the short pump pulse is not giving rise to rotation of the molecule which would affect the KER spectra.

Switching on the driving field leads to new structures in the spectra. At the smallest considered driving field intensity
[Figs. 3(c) and 3(d)] the spectra mostly follow the curves obtained in the absence of the driving field [marked with broken black lines from Fig. 3(c) onward], however, these serve only as a background on which a series of sharp resonances are superimposed. The asymmetric profile of these peaks indicates that they are Fano-type resonances [63-65]. 
TABLE I. Total excited population $\left(P_{\mathrm{ex}}\right)$ and its percentage dissociated $\left(P_{\mathrm{dis}}\right)$ by $t_{\text {final }}=1000 \mathrm{fs}$ for the different driving field intensities.

\begin{tabular}{|c|c|c|c|c|c|c|c|c|c|c|}
\hline \multirow[b]{2}{*}{$I_{d}\left(\mathrm{~W} / \mathrm{cm}^{2}\right)$} & \multicolumn{3}{|c|}{ Three-state (2D) } & \multicolumn{3}{|c|}{ Three-state (1D) } & \multicolumn{2}{|c|}{ Two-state (2D) } & \multicolumn{2}{|c|}{ Two-state (1D) } \\
\hline & $P_{\mathrm{ex}}^{\mathrm{tot}}$ & $P_{\mathrm{dis}}^{\mathrm{tot}}(\%)$ & $P_{\mathrm{dis}}^{i}(\%)\left(\begin{array}{l}\Sigma_{1} \\
\Pi_{1}\end{array}\right)$ & $P_{\mathrm{ex}}^{\mathrm{tot}}$ & $P_{\mathrm{dis}}^{\mathrm{tot}}(\%)$ & $P_{\mathrm{dis}}^{i}(\%)\left(\begin{array}{l}\Sigma_{1} \\
\Pi_{1}\end{array}\right)$ & $P_{\mathrm{ex}}^{\mathrm{tot}}$ & $P_{\mathrm{dis}}^{i}(\%)$ & $P_{\mathrm{ex}}^{\mathrm{tot}}$ & $P_{\mathrm{dis}}^{i}(\%)$ \\
\hline \multirow[t]{2}{*}{0} & 0.752 & 90.1 & 52.2 & 0.749 & 90.1 & 52.1 & 0.591 & 83.8 & 0.589 & 83.8 \\
\hline & & & 37.9 & & & 38.0 & & & & \\
\hline \multirow[t]{2}{*}{$1.69 \times 10^{12}$} & 0.749 & 88.8 & 50.8 & 0.750 & 88.9 & 50.8 & 0.592 & 79.6 & 0.589 & 79.8 \\
\hline & & & 38.0 & & & 38.1 & & & & \\
\hline \multirow[t]{2}{*}{$6.73 \times 10^{12}$} & 0.741 & 88.9 & 48.0 & 0.753 & 87.1 & 48.1 & 0.594 & 73.8 & 0.586 & 72.8 \\
\hline & & & 40.9 & & & 39.0 & & & & \\
\hline \multirow[t]{2}{*}{$2.68 \times 10^{13}$} & 0.698 & 87.7 & 51.8 & 0.762 & 65.7 & 34.6 & 0.598 & 80.9 & 0.574 & 45.9 \\
\hline & & & 35.9 & & & 31.1 & & & & \\
\hline \multirow[t]{2}{*}{$4 \times 10^{13}$} & 0.662 & 89.5 & 54.5 & 0.766 & 58.1 & 31.2 & 0.598 & 82.3 & 0.565 & 33.0 \\
\hline & & & 35.0 & & & 26.9 & & & & \\
\hline
\end{tabular}

The validity of the Fano theory in our investigation can be best understood by considering the dressed-state picture described is Sec. II B. Looking at Fig. 2 it can be seen that the upper adiabatic surface, $V_{2}$ for the two-state and $V_{3}$ for the three-state description (the adiabatic surfaces are indexed in order of their energies at the Franck-Condon region), constitutes the bound state embedded in the continuum of the lower adiabatic states necessary for the Fano theory.

Focusing first on the simpler two-state model, an inspection of the time evolution of the nuclear probability density shows that for the smallest considered driving field intensity, when the coupling between the electronic states is small, the system's behavior is mostly diabatic. That is, most of the excited wave packet follows the dynamics showed in the absence of a driving field. Nevertheless, part of the wave packet gets transferred to the $V_{2}$ adiabatic upper state. On Fig. 2(b) we can see that the two adiabatic surfaces form a light-induced avoided crossing or a light-induced conical intersection in case of the 1D and 2D descriptions, respectively. As the wave packet on the $V_{2}$ potential oscillates, at each passage over the LIAC (LICI) region it bifurcates. In the outward moving phase (toward larger $R$ values), part of the wave packet is transferred to the dissociating region of the lower adiabatic surface $V_{1}$ (i.e., $V_{\Sigma_{2}}$ ) from where it follows the usual path through the intrinsic avoided crossing to finally dissociate on $V_{\Sigma_{1}}$. The LIAC (LICI) is formed in the vicinity of $R_{\mathrm{LICI}}=2.68 \AA$ and $\theta=\pi / 2$, nevertheless, at this moderate driving field intensity the energy gap between the adiabatic surfaces is small for all $\theta$ values (at $R_{\mathrm{LICI}}$ ), which has multiple consequences. On one hand, the population transfer to the dissociating part of $V_{1}$ can take place at all angles, although with reduced efficiency. This means that after multiple passages of the oscillating wave packet through the coupling region, only a small part of the population remains trapped in the $V_{2}$ potential. This can be observed in the small reduction of the dissociation yield at $t_{f}=1000$ fs compared to the "no driving field" case as shown in Table I, which presents the amount of the excited population in the system along its percentage that dissociated by the end of the calculations. On the other hand, the curvature of $V_{2}$ in the direction of $\theta$ is small, therefore, it is unable to guide the wave packet toward the LICI where the population transfer would be more efficient. In other words, the weak driving field is unable to rotate the molecule, hence there is no noticeable difference between the $1 \mathrm{D}$ and $2 \mathrm{D}$ results. The structure of the resonances resembles the vibrational spectra of $V_{2}$, however, the resonance energies do not coincide with the vibrational eigenvalues. This is attributed to the energy shift produced by the configuration interaction between the bound and continuum states [63-65]. As the calculation of these energy shifts is a labor-intensive task and our aim is not to reproduce the KER spectra by using the Fano theory, we will not pursue to exactly predict the position of the resonances.

For the three-state model, similarly to the two-state case, the system's behavior for the smallest driving field intensity remains diabatic. The difference with the inclusion of the $\Pi$ state is the formation of two additional crossings around $R_{\mathrm{LICI}}=2.67 \AA$ and $\theta \in\{0, \pi\}$ [see Fig. 2(c)]. In the region of these LIACs or LICIs, the lower two adiabatic surfaces show a strong mixing of all three states [as illustrated by the coloring of Figs. 2(a) and 2(c), albeit for a different driving field intensity], which facilitates a population transfer between them. Accordingly, the wave packet on the $V_{3}$ surface may also dissociate on the $\Pi_{1}$ state. This is confirmed by the KER spectra, as the Fano resonances appear at the same energies for both the $\Sigma_{1}$ and the $\Pi_{1}$ results. They also coincide with the resonances obtained for the two-state model, which is understandable as the upper surface $V_{3}$ is mostly formed from the $\Sigma_{1}$ and $\Sigma_{2}$ states as in the two-state model (see Fig. 2).

Increasing the driving field intensity to $I_{d}=6.73 \times$ $10^{12} \mathrm{~W} / \mathrm{cm}^{2}$, one can see on Figs. 3(e) and 3(f) that the Fano resonances remain the dominant features of the spectra. Higher intensity means stronger coupling between the electronic states, but the behavior of the system still remains closer to the diabatic limit. This is suggested by the fact that the dissociation yields are still fairly close to the no driving field case. Compared to the previous case, the effect of the increased intensity is reflected in the shift and the broadening [66] of the resonances. Also, small discrepancies appear between the 1D and 2D calculations around $1.1 \mathrm{eV}$. New peaks appear in the 2D results, which indicates that rotational modes have been excited.

At the next intensity, $I_{d}=2.68 \times 10^{13} \mathrm{~W} / \mathrm{cm}^{2}$, the $1 \mathrm{D}$ and 2D results differ significantly, although the resonances can still be observed for both cases. For the 1D model there is a momentous drop in the dissociation yield (see Table I). In the three-state calculations this is more pronounced for $\Sigma_{1}$. This can be explained as follows: the system starts to 
behave adiabatically, that is, a larger part of the excited wave packet follows the adiabatic surfaces. Also, due to the higher intensity the shape of these surfaces become more distorted, that is, the energy gap between $V_{2}-V_{1}$ (two-state) or $V_{3}-V_{2}$ (three-state) widens along the $\theta$ direction far away from the LIAC (LICI) regions at $\pi / 2$ (see Fig. 2). As a result, the population transfer to the lower surfaces becomes less efficient and the population remains trapped on the upper adiabatic surface. However, this bond hardening effect is not present in the 2D results. In contrast, an increase of the dissociation yield on the $\Sigma_{1}$ state can be observed. This is the result of the driving-field-induced rotation of the molecule, which guides the trapped wave packet toward the LICI position where the population transfer to the dissociating states can take place with the highest efficiency. Also, the rotational fine structure observed for the previous intensity becomes more apparent. For the highest considered intensity, these trends become even more pronounced. In 1D a larger part of the population remains trapped, while in the $2 \mathrm{D}$ situation the dissociation rate almost reaches the values obtained without driving field.

Looking at Table I, it is worth mentioning that the applied driving field modifies not only the dissociation rate of the molecule, but also the efficiency of the pump process. Accordingly, for the two-state 1D model we see a small decrease of the excited population with the increase of the driving field intensity. This can be attributed to a similar bond hardening effect than the one described earlier. In 2D this effect is compensated by a weak alignment of the molecule with the driving field which facilitates the population transfer, hence resulting in a minor increase of the excited populations. Explaining the three-state results is far from being trivial due to the aforementioned multiphoton processes. Interestingly, in this case the $1 \mathrm{D}$ excited populations increase with the pump intensity, while the 2D ones decrease. Again, the change in the $2 \mathrm{D}$ population is related to the alignment of the molecule, which although amplifies the population transfer to the $\Pi_{1}$ state, it also reduces the transfer to the $\Sigma_{2}$ state leading to an overall decrease.

\section{B. Angular distribution}

Attention now turns to the angular distribution of the dissociation products. The results are presented on Fig. 4 with the three-state calculations being shown in the left and the two-state ones in the right columns. Similarly to Fig. 3, the different rows correspond to the employed various driving field intensities in increasing order. Each panel exhibits the distributions obtained from the 2D and 1D calculations with continuous and dashed lines, respectively.

Starting with the two-state situation without the driving field [Fig. 4(b)] we can see that the photofragments are ejected predominantly in the polarization direction of the pump pulse, which is expected, since the transition dipole moment that promotes the dissociating wave packet to the excited state $\left(\mu_{\Sigma_{1} \Sigma_{2}}\right)$ is parallel to the molecular axis. The curves, however, deviate from a $\cos ^{2} \theta$ shape (characteristic to single-photon excitations and TDMs parallel with the molecular axis) indicating that we are in the nonlinear regime with the pump intensity and that multiphoton processes are to be expected. Also, there is a small deviation between the 1D and 2D distributions. This can be attributed to the fact that the pump pulse tends to align the molecules along its polarization axis owing to the nonvanishing permanent dipole moments. This alignment is described only in the 2D model, which considers the rotation of the molecule as well, and leads to higher dissociation probability in the direction of the field for the 2D model. Around the perpendicular direction, the opposite effect is valid. However, as we used a short pump pulse, this is not too pronounced.

When the $\Pi_{1}$ state is included in the calculations [Fig. 4(a)], part of the initial population is transferred to this state. The corresponding TDM $\left(\mu_{\Sigma_{1} \Pi_{1}}\right)$ is perpendicular to the molecular axis, therefore, the wave packet on this state dissociates in the perpendicular direction to the polarization axis of the pump pulse. The fingerprint of the population mixing due to multiphoton processes mentioned in the previous section can be identified as part of the population around the angle $\theta=\pi / 4$ is transferred from the $\Sigma_{2}$ to the $\Pi_{1}$ state, resulting in a broader and flatter distribution for $\Pi_{1}$ than the $\sin ^{2} \theta$ shape expected in case of single-photon transitions, and a reduction in the same region for the angular distribution on $\Sigma_{1}$. Only at the $\theta=0$ and $\pi$ angles in the $1 \mathrm{D}$ description does $P_{\text {ang }}^{\Sigma_{1}}(\theta)$ reach its value from the two-state scenario, where the dipole coupling with the $\Pi$ state is zero. In the $2 \mathrm{D}$ case, the alignment effect is emphasized even more than on Fig. 4(b). This is not surprising since the population promoted to the $\Pi_{1}$ state is perpendicular to the laser polarization direction providing a more efficient way to rotate the molecule despite its smaller PDM. An unexpected feature of the 2D results is the nonvanishing $P_{\text {ang }}^{\Pi_{1}}$ at the $\theta=0$ and $\pi$ angles despite the zero dipole coupling. This effect was recently observed for another diatomic system with TDM perpendicular to the molecular axis [67], and it can be explained as follows: Similarly to the description given for the effect of a driving field while discussing the KER spectra, the interaction with the pump pulse leads to LICIs at the $\theta=0$ and $\pi$ angles between the $\Sigma_{1}-\Pi_{1}$ potential surfaces, although at different $R$ value as the pump energy is different. The induced nonadiabatic effect provides an efficient route for the population transfer to the $\Pi_{1}$ state at these angles.

Switching on the driving field, we can see on Figs. 4(c) and 4(d) that no major changes appear in the shape of the angular distributions for the smallest considered intensity. The only difference compared to the no driving field case, in accordance with the data of Table I, is a modest overall drop of the dissociation yield on the $\Sigma_{1}$ state both in the 1D and 2D models. This effect is more pronounced around the $\theta=0$ and $\pi$ angles, while the dissociation yield on the $\Pi_{1}$ state for the three-state calculations remains mostly unaffected. This is consistent with the discussion given at the previous section, namely, a trapping effect occurs on the upper adiabatic LIP as the energy gap between the potential surfaces widens away from the LICI and LIAC regions at $\theta=\pi / 2$.

Increasing the intensity to $I_{d}=6.73 \times 10^{12} \mathrm{~W} / \mathrm{cm}^{2}$ [Figs. 4(e) and 4(f)] further accentuates the trapping effect, especially for the two-state calculations. More importantly, the behavior of the $1 \mathrm{D}$ and 2D models starts to differ [9]. The stronger driving field is now able to slightly rotate the wave packet on the upper adiabatic surface toward the LICI 


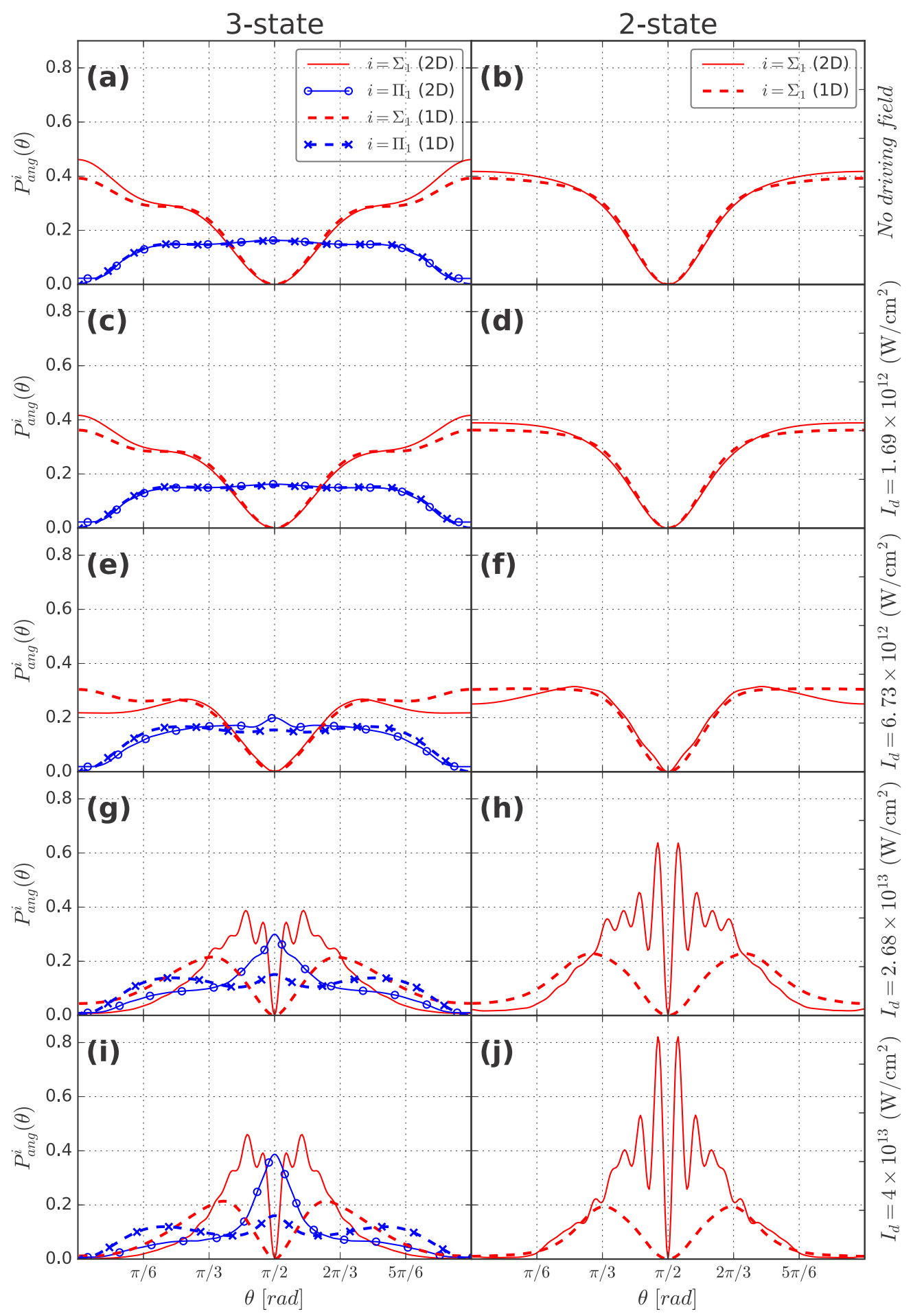

FIG. 4. Angular distribution of the photofragments for the considered driving field intensities (indicated on the right axis of each row). The 1D and 2D results are represented with dashed and continuous red lines for the $\Sigma_{1}$, while dashed and continuous blue lines with $\times$ and $\circ$ symbols for the $\Pi_{1}$ electronic state.

position where it can decay to the lower surface(s). This leads in $2 \mathrm{D}$ to a reduction of the dissociation probability along the polarization direction of the driving field and to an increase in the perpendicular direction. In the three-state calculation, this increase is observed on both dissociating channels.

At $I_{d}=2.68 \times 10^{13} \mathrm{~W} / \mathrm{cm}^{2}$ [Figs. $4(\mathrm{~g})$ and $4(\mathrm{~h})$ ], the angular distributions become more structured for all models.
In $1 \mathrm{D}$ the bond hardening continues to dominate along the polarization direction, however, this effect is counterweighted by the stronger nonadiabatic coupling between the electronic states around the LIAC position $(\theta=\pi / 2)$. At intermediate angles, these two effects balance out each other leading to the two broad peaks in the angular distribution on the $\Sigma_{1}$ state. It was mentioned in the previous section that at this intensity 
the system starts to behave adiabatically. Accordingly, in the three-state scenario, part of the $\Pi_{1}$ population is transferred to $\Sigma_{2}$ [see $V_{2}$ on Figs. 2(a) and 2(c)] and later dissociates on $\Sigma_{1}$. This is, however, offset by the two LIACs at $\theta=0$ and $\pi$ resulting in the undulated angular distribution on $\Pi_{1}$. In the $2 \mathrm{D}$ scheme, aside from the processes presented for $1 \mathrm{D}$ one, we also have the rotation. At this intensity, the driving field guides the wave packet on the upper LIP toward the LICI with higher efficiency than previously, offsetting almost completely the bond hardening effect. Meanwhile, many rotational modes are excited. As our initial wave function had $J=0$, according to the selection rules, the excited wave-packet components on $\Sigma_{2}$ have odd $J$ values. The spiky structure in the angular distribution on $\Sigma_{1}$ is actually the fingerprint of the interference produced by these wave-packet components. Owing to the symmetry properties of the Legendre polynomials, they interfere destructively at $\theta=\pi / 2$. For the three-state model, a similar interference effect is observed on the $\Pi_{1}$ state. However, in this case the wave packet has even $J$-value components resulting in a constructive interference around $\theta=\pi / 2$. At the highest considered driving field intensity, the angular distribution is shaped by the same processes, but with greater impact, resulting in similar distributions.

\section{CONCLUSIONS}

In summary, we can say that the complementary information offered by the kinetic energy release spectra and the angular distribution of the photofragments provides a more comprehensive picture about the behavior of the LiF molecule under the action of external laser fields. The inclusion of the $\Pi_{1}$ electronic state in the calculation proved to have a significant impact on all physical quantities examined. First of all, the total amount of excited population in the system increased, while the excited population on the $\Sigma_{1}$ state decreased slightly due to population mixing induced by multiphoton processes. The presence of the $\Pi_{1}$ state also modified the energy and the angular distribution of the photofragments. For this reason, we believe that it must be included in any theoretical approach aspiring to produce results comparable with experimental data.

We saw that by placing the molecule in an off-resonant continuous wave driving field may influence heavily its dynamics. First of all, it affects the efficiency of pumping the system from its initial ground state to the excited ones. More importantly, the KER spectra are shaped by two effects, namely, Fano resonances and bond hardening. Both processes were explained with the help of the lightinduced potentials that originate from the interaction with this driving field. Accordingly, part of the wave packet remains temporarily trapped on the upper adiabatic LIP. The Fano resonances originate from the configuration interaction between the bound states that constitutes this trapped wave packet with the continuum of the lower LIPs. We also found that important differences developed between the 1D and 2D descriptions with the increase of the driving field intensity. For the 1D calculations, the trapping effect became more dominant, leading to a considerable drop of the dissociation yield. In contrast, this bond hardening was not present in $2 \mathrm{D}$ as the driving field rotated the wave packet toward the LICI position, hence facilitating the population transfer to the lower surfaces and consequently the dissociation. In this case, new peaks appeared in the spectra owing to the excitation of rotational modes.

The fingerprints of these processes were also observed in the angular distribution. The trapping effect manifested in a continuous decrease of the dissociation probability with increasing intensity along the polarization direction of the external laser field. For the three-state calculations, this effect was more pronounced for the $\Sigma_{2}$ state. At the highest considered intensities, the rotation of the molecule described in $2 \mathrm{D}$ gave rise to interferences between the wave-packet components with different $J$ values both on the $\Sigma_{1}$ and $\Pi_{1}$ states in case of the three-state calculations.

We are hopeful that our findings attract attention from the community and stimulate some much needed experiments that would confirm the importance of both the $\Pi_{1}$ state and the rotation in an adequate theoretical approach.

\section{ACKNOWLEDGMENTS}

This research was supported by the EU-funded Hungarian Grant No. EFOP-3.6.2-16-2017-00005, and the ELI-ALPS Project No. GINOP 2.3.6-15-2015-00001. We are grateful to NKFIH for financial support (Grant No. K128396). The supercomputing service of NIIF has been used for this work.
[1] J. L. Krause, K. J. Schafer, and K. C. Kulander, Phys. Rev. Lett. 68, 3535 (1992).

[2] M. Lewenstein, P. Balcou, M. Y. Ivanov, A. L'Huillier, and P. B. Corkum, Phys. Rev. A 49, 2117 (1994).

[3] K. J. Schafer, B. Yang, L. F. DiMauro, and K. C. Kulander, Phys. Rev. Lett. 70, 1599 (1993).

[4] A. D. Bandrauk and M. L. Sink, Chem. Phys. Lett. 57, 569 (1978).

[5] E. E. Aubanel, J.-M. Gauthier, and A. D. Bandrauk, Phys. Rev. A 48, 2145 (1993).

[6] A. D. Bandrauk, E. E. Aubanel, and J.-M. Gauthier, in Molecules in Laser Fields, edited by A. D. Bandrauk (Marcel Dekker, New York, 1994).
[7] P. H. Bucksbaum, A. Zavriyev, H. G. Muller, and D. W. Schumacher, Phys. Rev. Lett. 64, 1883 (1990).

[8] A. Zavriyev, P. H. Bucksbaum, H. G. Muller, and D. W. Schumacher, Phys. Rev. A 42, 5500 (1990).

[9] R. Numico, A. Keller, and O. Atabek, Phys. Rev. A 52, 1298 (1995).

[10] S. Chelkowski, T. Zuo, O. Atabek, and A. D. Bandrauk, Phys. Rev. A 52, 2977 (1995).

[11] V. N. Serov, A. Keller, O. Atabek, and N. Billy, Phys. Rev. A 68, 053401 (2003).

[12] F. Anis and B. D. Esry, Phys. Rev. A 77, 033416 (2008).

[13] J. H. Posthumus, Rep. Prog. Phys. 67, 623 (2004). 
[14] G. J. Halász, A. Csehi, Á. Vibók, and L. S. Cederbaum, J. Phys. Chem. A 118, 11908 (2014).

[15] W. R. Anderson, B. M. Wilson, R. C. Ormerod, and T. L. Rose, J. Chem. Phys. 74, 3295 (1981).

[16] J. A. Silver, D. R. Worsnop, A. Freedman, and C. E. Kolb, J. Chem. Phys. 84, 4378 (1986).

[17] O. Vasyutinskii, K. Korovin, and B. Picheev, Opt. Spectrosc. 82, 171 (1997) [Optika i Spektroskopiya 82, 191 (1997)].

[18] K. O. Korovin, A. A. Veselov, O. S. Vasyutinskii, and D. Zimmermann, Opt. Spectrosc. 93, 530 (2002) [Optika i Spektroskopiya 93, 577 (2002)].

[19] K. O. Korovin, A. A. Veselov, E. M. Mikheev, O. S. Vasyutinskii, and D. Zimmermann, Opt. Spectrosc. 99, 880 (2005) [Optika i Spektroskopiya 99, 917 (2005)].

[20] H. H. Telle and A. J. Tambini, J. Phys. B: At., Mol. Opt. Phys. 22, L315 (1989).

[21] L. J. Collier and H. H. Telle, Rapid Commun. Mass Spectrom. 7, 519 (1993).

[22] K. O. Korovin, E. Heinecke, A. Patzer, T. Liebig, O. S. Vasyutinskii, and D. Zimmermann, Eur. Phys. J. D 44, 57 (2007).

[23] S. De, M. Magrakvelidze, I. A. Bocharova, D. Ray, W. Cao, I. Znakovskaya, H. Li, Z. Wang, G. Laurent, U. Thumm, M. F. Kling, I. V. Litvinyuk, I. Ben-Itzhak, and C. L. Cocke, Phys. Rev. A 84, 043410 (2011).

[24] M. Magrakvelidze, C. M. Aikens, and U. Thumm, Phys. Rev. A 86, 023402 (2012).

[25] C. Wunderlich, E. Kobler, H. Figger, and T. W. Hänsch, Phys. Rev. Lett. 78, 2333 (1997).

[26] K. Sändig, H. Figger, and T. W. Hänsch, Phys. Rev. Lett. 85, 4876 (2000).

[27] A. Csehi, G. J. Halász, L. S. Cederbaum, and Á. Vibók, J. Phys. Chem. Lett. 8, 1624 (2017).

[28] A. Csehi, G. J. Halász, L. S. Cederbaum, and Á. Vibók, Phys. Chem. Chem. Phys. 19, 19656 (2017).

[29] H. Köppel, W. Domcke, and L. S. Cederbaum, Multimode Molecular Dynamics Beyond the Born-Oppenheimer Approximation, Advances in Chemical Physics (Wiley, Hoboken, NJ, 2007), pp. 59-246.

[30] M. Baer, Phys. Rep. 358, 75 (2002).

[31] G. A. Worth and L. S. Cederbaum, Annu. Rev. Phys. Chem. 55, 127 (2004).

[32] W. Domcke, D. R. Yarkony, and H. Köppel, Conical Intersections: Electronic Structure, Dynamics \& Spectroscopy, Advanced Series in Physical Chemistry (World Scientific, Singapore, 2004).

[33] M. Baer, Beyond Born-Oppenheimer: Electronic Nonadiabatic Coupling Terms and Conical Intersections (Wiley, Hoboken, NJ, 2006).

[34] G. J. Halász, Á. Vibók, M. Šindelka, N. Moiseyev, and L. S. Cederbaum, J. Phys. B: At., Mol. Opt. Phys. 44, 175102 (2011).

[35] G. J. Halász, M. Šindelka, N. Moiseyev, L. S. Cederbaum, and Á. Vibók, J. Phys. Chem. A 116, 2636 (2012).

[36] G. J. Halász, Á. Vibók, N. Moiseyev, and L. S. Cederbaum, J. Phys. B: At., Mol. Opt. Phys. 45, 135101 (2012).

[37] G. J. Halász, Á. Vibók, N. Moiseyev, and L. S. Cederbaum, Phys. Rev. A 88, 043413 (2013).

[38] G. J. Halász, Á. Vibók, and L. S. Cederbaum, J. Phys. Chem. Lett. 6, 348 (2015).
[39] A. Natan, M. R. Ware, V. S. Prabhudesai, U. Lev, B. D. Bruner, O. Heber, and P. H. Bucksbaum, Phys. Rev. Lett. 116, 143004 (2016).

[40] Z. Sun, C. Wang, W. Zhao, and C. Yang, J. Chem. Phys. 149, 224307 (2018).

[41] P. V. Demekhin and L. S. Cederbaum, J. Chem. Phys. 139, 154314 (2013).

[42] J. Kim, H. Tao, J. L. White, V. S. Petrović, T. J. Martinez, and P. H. Bucksbaum, J. Phys. Chem. A 116, 2758 (2012).

[43] M. E. Corrales, J. González-Vázquez, G. Balerdi, I. R. Solá, R. de Nalda, and L. Bañares, Nat. Chem. 6, 785 (2014).

[44] A. Tóth, P. Badankó, G. J. Halász, Á. Vibók, and A. Csehi, Chem. Phys. 515, 418 (2018).

[45] H. Werner and W. Meyer, J. Chem. Phys. 74, 5802 (1981).

[46] H. Nakamura and D. G. Truhlar, J. Chem. Phys. 117, 5576 (2002).

[47] A. D. Bandrauk and J. M. Gauthier, J. Phys. Chem. 93, 7552 (1989).

[48] A. D. Bandrauk and J.-M. Gauthier, J. Opt. Soc. Am. B 7, 1420 (1990).

[49] P. Marquetand, C. Meier, and V. Engel, J. Chem. Phys. 123, 204320 (2005).

[50] C.-C. Shu, K.-J. Yuan, D. Dong, I. R. Petersen, and A. D. Bandrauk, J. Phys. Chem. Lett. 8, 1 (2017).

[51] S. Scheit, Y. Arasaki, and K. Takatsuka, J. Phys. Chem. A 116, 2644 (2012).

[52] S. Scheit, Y. Arasaki, and K. Takatsuka, J. Chem. Phys. 140, 244115 (2014).

[53] Y. Arasaki, Y. Mizuno, S. Scheit, and K. Takatsuka, J. Chem. Phys. 144, 044107 (2016).

[54] H.-J. Werner, P. J. Knowles, G. Knizia, F. R. Manby, M. Schütz, and others, MOLPRO, version 2015.1, a package of ab initio programs.

[55] A. J. C. Varandas, J. Chem. Phys. 131, 124128 (2009).

[56] J. F. Triana, D. Peláez, and J. L. Sanz-Vicario, J. Phys. Chem. A 122, 2266 (2018).

[57] A. Hofmann and R. de Vivie-Riedle, Chem. Phys. Lett. 346, 299 (2001).

[58] S. Chu, J. Chem. Phys. 75, 2215 (1981).

[59] S.-I. Chu and D. A. Telnov, Phys. Rep. 390, 1 (2004).

[60] H.-D. Meyer, U. Manthe, and L. Cederbaum, Chem. Phys. Lett. 165, 73 (1990).

[61] M. Beck, A. Jäckle, G. Worth, and H.-D. Meyer, Phys. Rep. 324, 1 (2000).

[62] G. A. Worth, M. H. Beck, A. Jäckle, and H.-D. Meyer, The MCTDH package, version 8.2 (University of Heidelberg, Heidelberg, 2000); H.-D. Meyer, The MCTDH package, version 8.3 and 8.4 (University of Heidelberg, Heidelberg, 2002 and 2007), http://mctdh.uni-hd.de

[63] U. Fano, Phys. Rev. 124, 1866 (1961).

[64] E. F. van Dishoeck, M. C. van Hemert, A. C. Allison, and A. Dalgarno, J. Chem. Phys. 81, 5709 (1984).

[65] S. Y. Grebenshchikov and D. Picconi, Chem. Phys. 515, 60 (2018).

[66] J. Wang, Non-Adiabatic Dinamics of Excited States of Molecular Oxigen, Ph.D. thesis, University of Adelaide, 1989.

[67] P. Badankó, G. J. Halász, L. S. Cederbaum, Á. Vibók, and A. Csehi, J. Chem. Phys. 149, 181101 (2018). 